\title{
Energy Conversion with Doubly Fed Machines
}

\author{
J. Bendl, M. Chomat and L. Schreier \\ Institute of Electrical Engineering \\ Academy of Sciences \\ Dolejškova 5, 18202 Prague 8 \\ phone:+420 266052 006, fax:+420 286890 433, e-mail: bendl@iee.cas.cz
}

\begin{abstract}
The presented paper deals with doubly fed machines (DFM) operating in units that convert mechanical to electric power and vice versa. We concentrate especially on their usage in pumped storage plants which, used in the power system, can increase its stability and operating efficiency. A mathematical model of the power unit with a doubly fed machine and a cycloconverter in its rotor circuit was derived. The model includes vector control of the rotor voltage which enables us to analyse both steady and transient states.
\end{abstract}

\section{Key words}

Energy conversion, doubly fed machines, vector control, transient phenomena.

\section{Introduction}

Electric power can be effectively accumulated by pumped storage plants. The principle of the accumulation is based on the fact that in periods of surplus power in the grid, the water is pumped up to the upper water reservoir and in periods, when there is a need to supply electric power into the grid, the water is let out of the tank through a turbine and its potential energy is transformed back to electric power.

Formerly this electric conversion was realized with standard synchronous machines that operated either as generators or as motors. The resulting pumping and turbine cycle carried out in this way reached overall efficiency less than $75 \%$. The pumping regime with a partial output was not acceptable due to hydraulic and economic reasons. It seems to be very favourable when the power unit with variable speed can be both in generator and in pumping operation. Even a small change of speed from the synchronism can increase the efficiency of the energy conversion by almost $10 \%$ and reduce the unfavourable effects of turbine cavitation. The use of frequency converter feeding the stator winding of a synchronous machine is only convenient for smaller power outputs. For larger outputs, almost 500MW can be considered, so called doubly fed machine (DFM) of induction type should be employed ([1], [2]). Its 3-phase stator winding is connected through a block transformer to the grid. The rotor of this machine has a 3-phase winding fed by variable frequencies from the frequency converter so that unit speed can be varied within the required range. As a frequency converter for large outputs, a 12-pulse cycloconverter fed from a separate transformer can be utilized.

Independent changes in speed and rotor voltage provide more freedom in control of the active and reactive power outputs of the machine both in motor and generator operation [3]. Moreover, this system, owing to its control that is faster than in systems with synchronous generators, is especially suitable for maintaining the stability of the power network.

A great advantage can be taken of a possibility of immediate output supply to the grid using the power of rotating masses at low decrease of speed. The cycloconverter can be also utilized for starting the unit.

The turbine is controlled so that it can operate at the optimal rotational speed for a particular vertical difference between the upper and lower levels in the water reservoirs. Other applications for DFMs are represented by generators in wind power plants, but they bring some specific problems.

\section{System under consideration}

The scheme of an adjustable-speed power unit with the DFM in pumped storage plants is shown in Fig. 1.

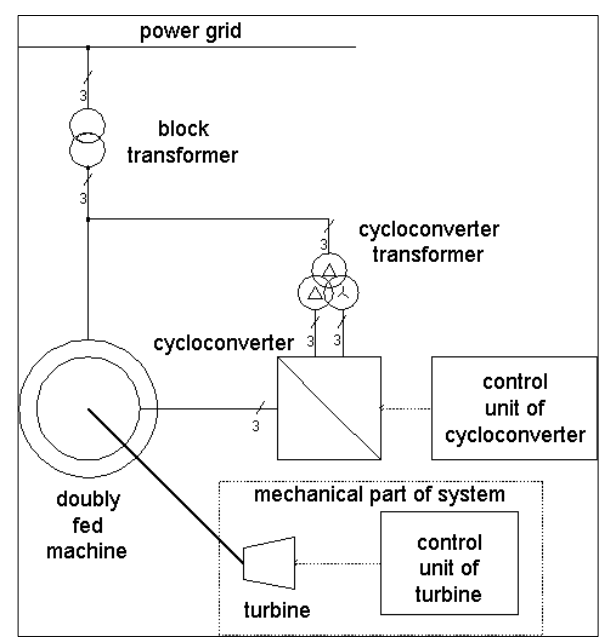

Fig. 1. Adjustable-speed power unit 
The stator of the DFM is connected to the power network through a block transformer. The rotor is driven by an adjustable-blade turbine and fed from a twelve-pulse cycloconverter with a three-winding transformer. The control circuitry is included in the system. At a given speed the rotor voltage (its amplitude and phase) should be controlled so that the demanded active and reactive power outputs can be reached, but neither stator nor rotor currents exceed permissible values given by the design of the machine. The phasors of the rotor voltage that meet these conditions define working regions of the DFM [4].

The operation of the unit can be analysed by means of a mathematical model. The model of the system consists of three parts: the model of the DFM, based on the space phasor theory, with the mechanical part of the system, the model of the 12-pulse cycloconverter with the transformer, which has been developed under the assumption of ideal switches, and the model of the control unit [5].

\section{Working Regions}

In the steady state, the DFM can be described by equations

$$
\begin{gathered}
\hat{\boldsymbol{U}}_{S} ? ? R_{S} ? j X_{S} ? \hat{\boldsymbol{I}}_{S} ? j X_{M} \hat{\boldsymbol{I}}_{R} \\
\hat{\boldsymbol{U}}_{R} ? j s X_{M} \hat{\boldsymbol{I}}_{S} ? ? R_{R} ? j s X_{R} ? \hat{\boldsymbol{I}}_{R}
\end{gathered}
$$

where $\hat{\boldsymbol{U}}_{S}, \hat{\boldsymbol{I}}_{S}$, and $\hat{\boldsymbol{U}}_{R}, \hat{\boldsymbol{I}}_{R}$ represent the phasors of the stator and rotor voltages and currents, $R_{S}, R_{R}, X_{S}, X_{R}$, and $X_{M}$ are the resistances and reactances of the machine, and $s$ is the slip. The above equations are only valid when the fundamental time and spatial harmonics of the machine quantities are taken into account. The solution of (1) and (2) may be written as

$$
\begin{gathered}
\hat{\boldsymbol{I}}_{S} ? \frac{? R_{R} ? j s X_{R} \hat{\boldsymbol{U}_{S}} ? j X_{M} \hat{\boldsymbol{U}}_{R}}{? R_{S} ? j X_{S} ? ? R_{R} ? j s X_{R} ? ? s X_{M}^{2}} \\
\hat{\boldsymbol{I}}_{R} ? \frac{? R_{S} ? j X_{S} ? \hat{\boldsymbol{U}}_{R} ? j s X_{M} \hat{\boldsymbol{U}}_{S}}{? R_{S} ? j X_{S} ? ? R_{R} ? j s X_{R} ? ? s X_{M}^{2}} .
\end{gathered}
$$

The active and reactive stator powers of the machine are

$$
\begin{aligned}
& P_{S} ? \frac{3}{2} \operatorname{Re} ? \hat{\boldsymbol{U}}_{S} \hat{\boldsymbol{I}}_{S}^{*} ? \\
& Q_{S} ? \frac{3}{2} \operatorname{Im} 2 \hat{\boldsymbol{U}}_{S} \hat{\boldsymbol{I}}_{S}^{*} ? \cdot
\end{aligned}
$$

The working regions of the DFM are given by the magnitude and phase shift of the rotor voltage phasor so that neither stator nor rotor currents exceed permissible values [4]. From (3) and (4) we can determine the relations for the boundaries of partial working regions limited by current amplitudes $I_{S M}$ and $I_{R M}$

$$
\begin{aligned}
& \hat{U}_{R} ? K_{1} ? K_{2} I_{S M} e^{j ? s} \\
& \hat{U}_{R} ? K_{3} ? K_{4} I_{R M} e^{j ?_{R}}
\end{aligned}
$$

where

$$
\begin{gathered}
K_{1} ? \frac{R_{R} ? j s X_{R}}{j X_{M}} U_{S} \\
K_{2} ? ? \frac{? R_{S} ? j X_{S} ? ? R_{R} ? j s X_{R} ? ? s X_{M}^{2}}{j X_{M}} \\
K_{3} ? \frac{j s X_{M}}{R_{S} ? j X_{S}} U_{S} \\
K_{4} ? \frac{? R_{S} ? j X_{S} ? ? R_{R} ? j s X_{R} ? ? s X_{M}^{2}}{R_{S} ? j X_{S}}
\end{gathered}
$$

The angles $f_{S}$ and $f_{R}$ vary from 0 to $2 p$ ?and the stator voltage phasor coincides with the real axis. The resulting working region is the intersection of both partial areas.

The partial regions are the circles with centres given by (9) and (11) with diameters determined by $I_{S M}$ and $I_{R M}$ together with (10) and (12).

The working regions of the DFM with a nominal power of $320 \mathrm{MW}$ and a nominal stator voltage of $22 \mathrm{kV}$ were computed for the control range of the rotor speed from 0.9 to 1.1 of the synchronous speed.

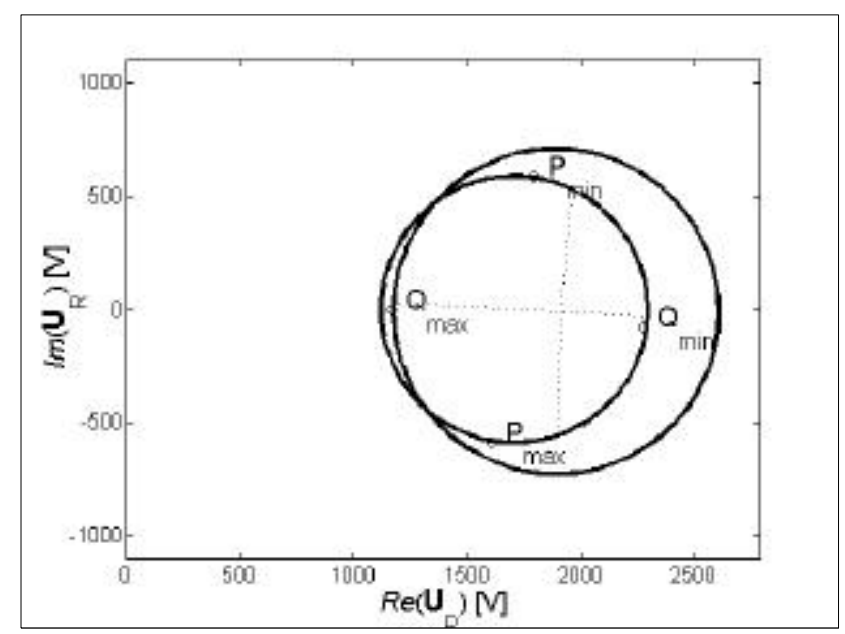

Fig. 2. Working regions of the DFM, $s=10 \%$

An example of the mechanical speed $10 \%$ below the synchronous speed is illustrated in the following figures. Fig. 2 shows the boundaries of the two partial working regions. The bigger circle corresponds to the limitation defined by the stator current and the smaller one to the limitation given by the rotor current. The rotor voltages supplied with the maximal or minimal active or reactive power by the machine are denoted by symbols $P_{\max }, P_{\min }$, $Q_{\max }$, and $Q_{\min }$. The dotted lines correspond to the operation with zero active or reactive power. The positions of the working regions in coordinates $P_{S^{-}} Q_{S}$ and $P_{R^{-}} Q_{R}$ are in Figs. 3 and 4 , where the isolines of the magnitude and angle of the rotor voltage are also depicted. 
Similar graphs can be defined for various speeds. Also demands for cyloconverter dimensation can be set regarding the requested range of regulation of the speed.

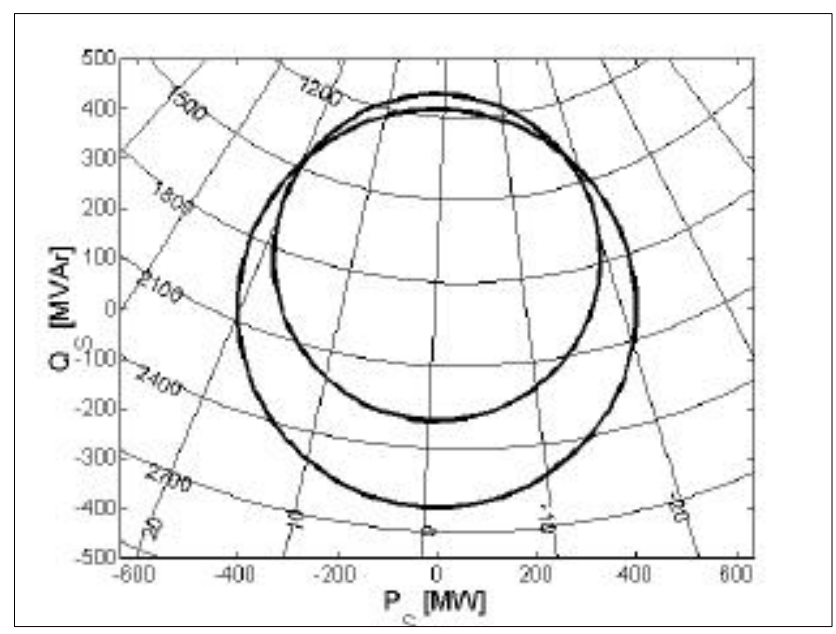

Fig. 3. Active and reactive powers of the stator, $s=10 \%$

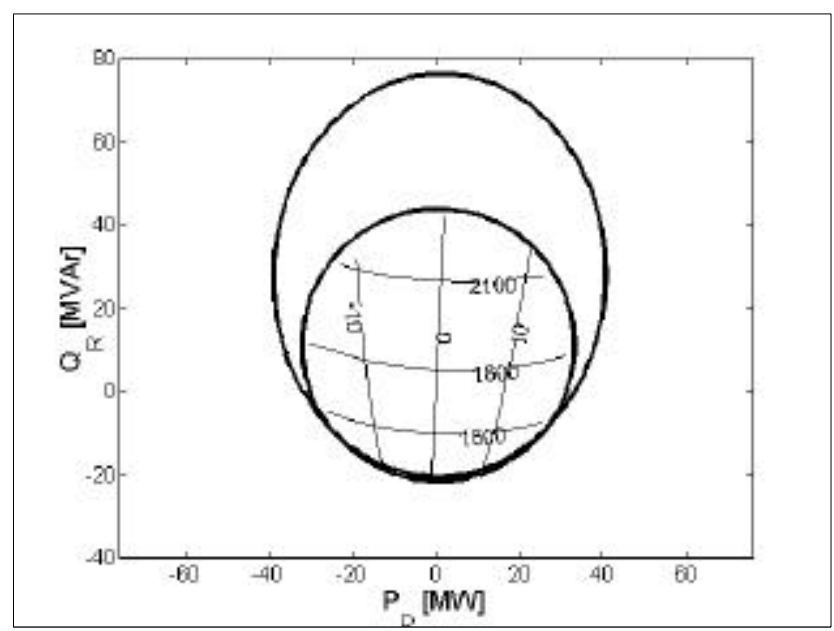

Fig. 4. Active and reactive powers of the rotor, $s=10 \%$

\section{Vector control}

The parameters of the power supplied to the power grid by the machine are controlled by the vector control [6]. The synchronous reference frame method of the vector control was chosen because it can control active and reactive powers generated by the stator directly. For this purpose, the equations of the DFM were transformed from the rotor co-ordinates $\mathrm{d}-\mathrm{q}$ to the synchronous coordinate system X-Y. The advantage of this approach is the fact that the X-Y components of the space phasor of the stator currents are proportional to the active and reactive powers of the stator.

The block diagram of the utilized control is in Fig. 5. Due to the fact that both the stator and rotor windings are accessible directly, no observer is needed to estimate the rotor currents. The demanded active and reactive stator powers are regulated by means of the output voltage of the cycloconverter, particularly by its amplitude and phase in the synchronous reference frame.

It is possible, in this way, to achieve a very fast response of the system to control signals, even in the case of a sudden change of the active power. An increase of the generated active power can temporarily be covered at the expense of the kinetic energy of the unit until the turbine control takes over. This approach necessitates, however, unit operation with some standby reserve in mechanical speed and it needs to be taken into account during the design of the system.

A specific problem is the regulation of the turbine speed so that it may work with maximum efficiency and unfavourable effect of cavitation can be minimized. These optimal speed depend on difference between the levels in the upper and lower water reservoirs of the pumped storage plant. Relation between the optimal speed and the output of the machine can be respected in the mathematical model of the power unit.

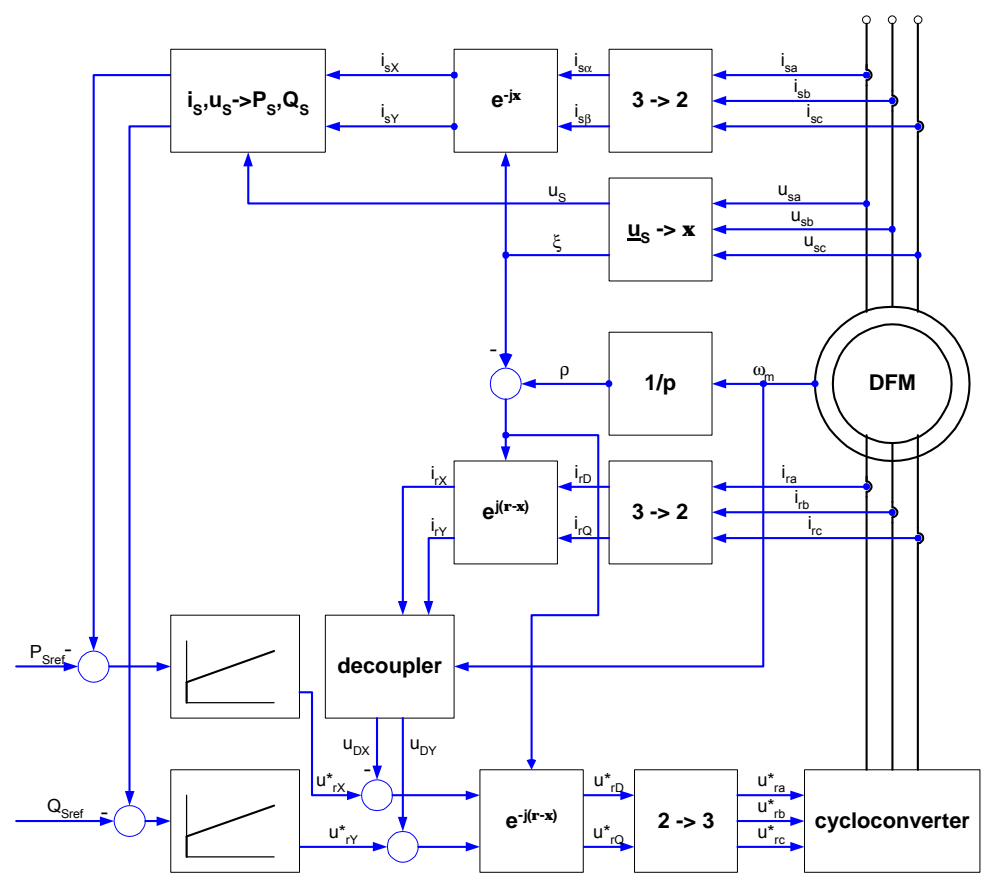

Fig. 5. Scheme of vector control 


\section{Extended vector control}

The existing results concern DFM with quite large outputs and with the cycloconverter in the rotor circuit connected to the grid over the transformer. Some cases, however, may occur when the rotor of DFM is fed from the voltage converter. Here machines of low outputs are concerned which can work under so called island operation or can be connected to the power grid with relatively long cables. Subsequently in these machines, due to unsymmetrical loads or failures, unsymmetrical voltages on stator terminals may arise. In the works [7], [8] so called modified method of the vector control was introduced, by means of which even at unsymmetrical stator voltage, the symmetrical current system can be reached. In these methods both of the components are controlled: the positive-sequence component of the stator currents like in the original vector control, and the negative-sequence component.

A simplified scheme of such a controller is in Fig. 6. Having transformed the stator currents into the reference frame rotating with the speed equal to the synchronous value, but in the opposite direction, we can regulate the negative sequence of the stator currents as DC quantities, which enables us to utilize PI controllers. The signals from both parts of the controller, positive-sequence and negative-sequence signals, are added to each other before being fed to the converter as the demanded values of the rotor voltage.

In Figs. 7 and 8 courses of stator currents are shown in the situation when $10 \%$ of the reverse component of the terminal voltage of the stator occurs suddenly during the operation. The Fig. 7 only presents the courses at control of the symmetrical component and Fig. 8 at control of both positive-sequence and negative-sequence components.

The mechanical speed is kept constant and equal to the synchronous speed during the transient process for the sake of simplicity. The vector controller maintains constant active power 1 p.u. and zero reactive power generated by the stator. The converter was considered for ideal in the model.

\section{Numerical simulation}

\section{A. Steady-state operation}

Simulation results of the steady-state operation of the system in Fig. 1 with 12-pulse cycloconverter are presented to illustrate the function of the developed numerical model. For example, the operation at speed 5\%

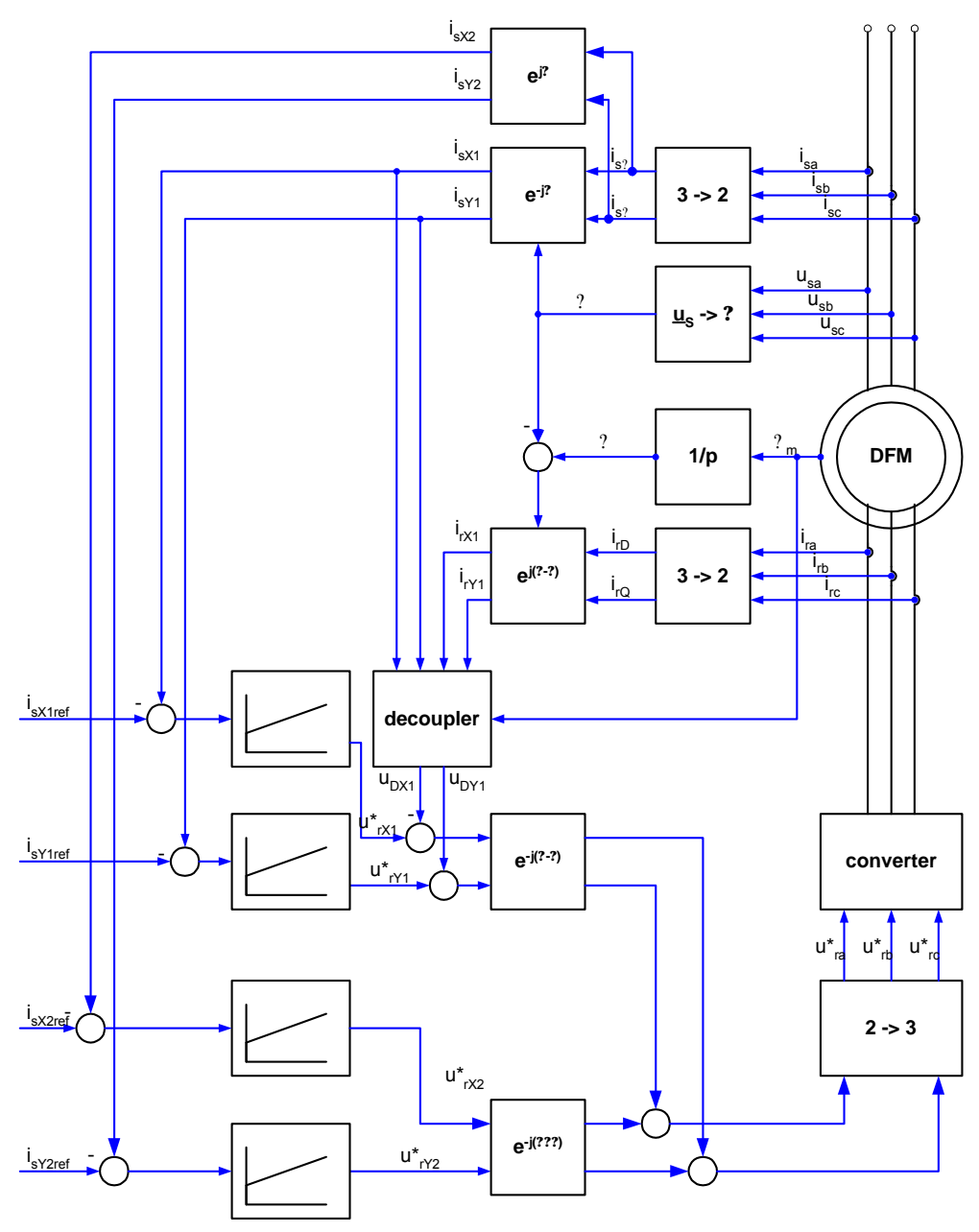

Fig. 6. Modified vector controller 
above the synchronous one with the maximum consumed active power was simulated. Fig. 9 shows the waveforms of the rotor voltage and current, stator current, and electromagnetic torque in the air gap of the machine. The presented quantities correspond to the actual waveforms of the phase voltages and currents in the rotor and stator.

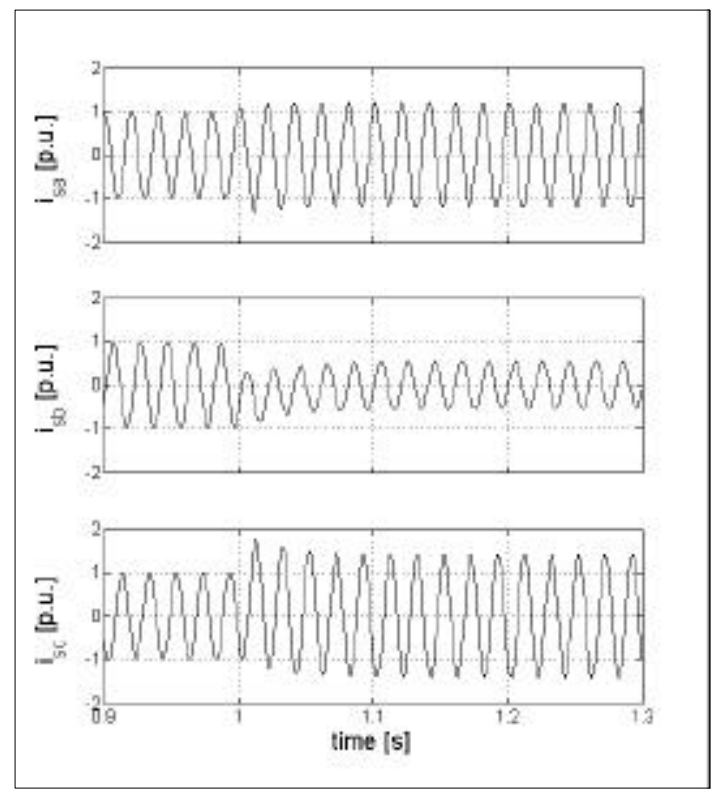

Fig. 7. Stator currents in individual phases for $10 \%$ negative-sequence voltage applied - conventional controller

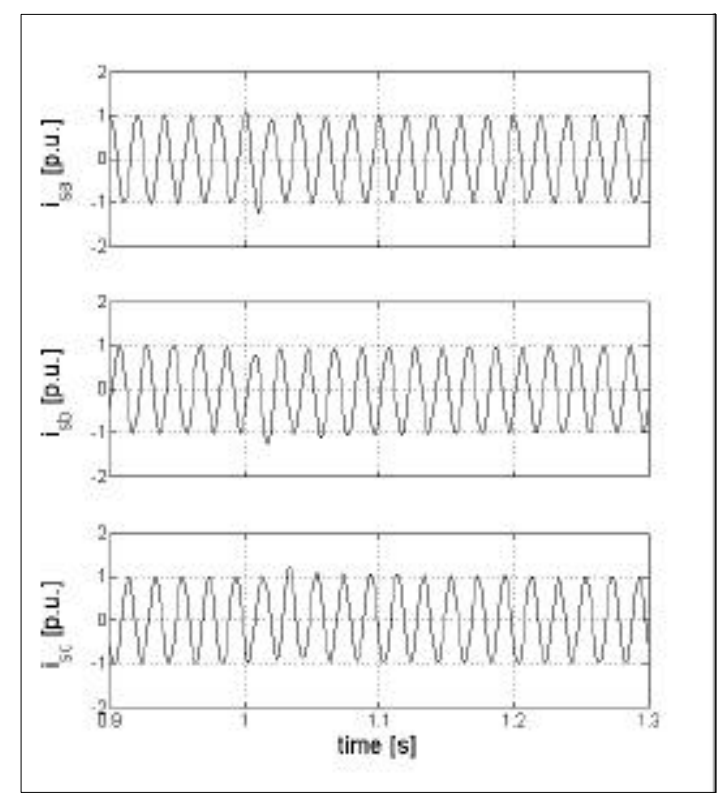

Fig. 8. Stator currents in individual phases for $10 \%$ negative-sequence voltage applied - modified controller

The results of the frequency analysis of the rotor voltage and current and stator current are shown in Fig. 10. The fundamental frequency of the rotor and stator quantities are $2.5 \mathrm{~Hz}$ and $50 \mathrm{~Hz}$, respectively. The magnitudes of higher harmonics are rated to the fundamental ones. The rotor voltage contains a wide spectrum of harmonics. Along with the fundamental harmonic the harmonics of the orders 5, 7, 59, 61, 179, and 181 are the most salient. Practically the rotor currents only contain the higherorder waves of orders $5,7,59$, and 61 . The stator currents contain the waves with both lower and higher order than the order of the fundamental wave.

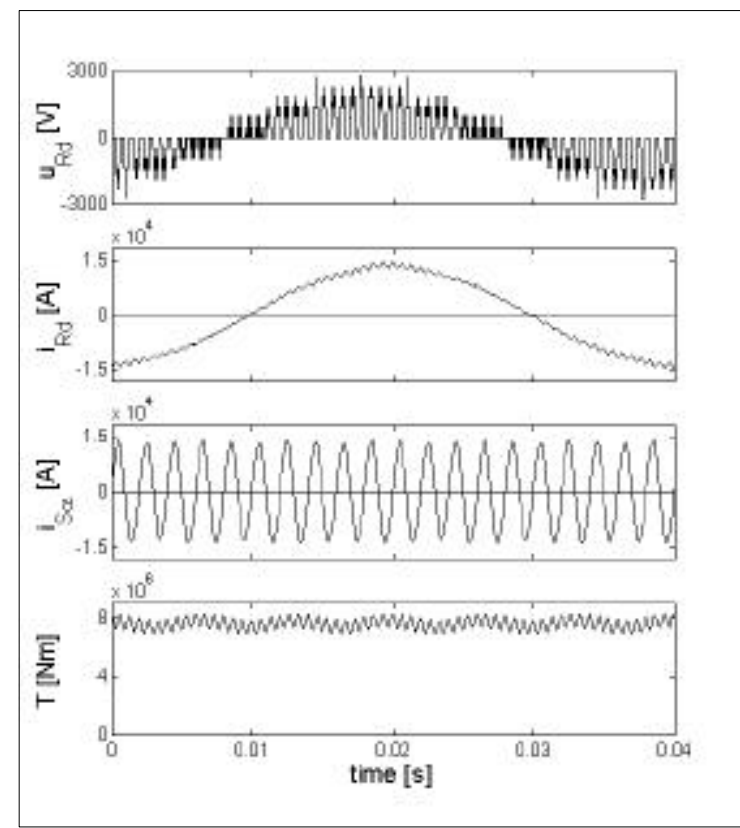

Fig. 9. Rotor voltage and current, stator current, and electromagnetic torque at steady state

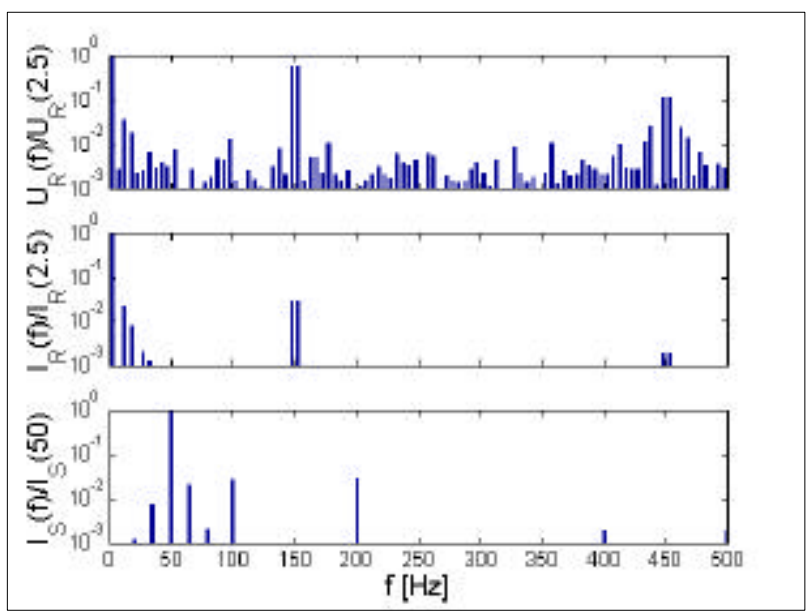

Fig. 10. Frequency analysis of rotor voltage and current, and stator current

\section{B. Transient operation}

The model of power unit enables solving of various transient states that may occur in operation. As an example, let us set transient states at the demanded load change.

The operation of the system at a change of the generated power from 300 to $200 \mathrm{MW}$ when the speed of the turbine is regulated to the optimal value is illustrated in Figs. 11-13. Fig. 11 shows the rotor voltage and current in the direct axis corresponding to the actual phase quantities. The amplitudes of the stator voltage and current are in Fig. 12. The resulting time developments of the active and reactive powers together with the mechanical angular speed are shown in Fig. 13.

The turbine regulator sets the lower, optimal, speed of the turbine for the new value of the demanded active power. 
The turbine control is fast enough to compensate for the decrease in the generated active power, otherwise the difference between the electric power generated by the machine and mechanical power output of the turbine would be covered at the expense of the inertial energy stored in the system.

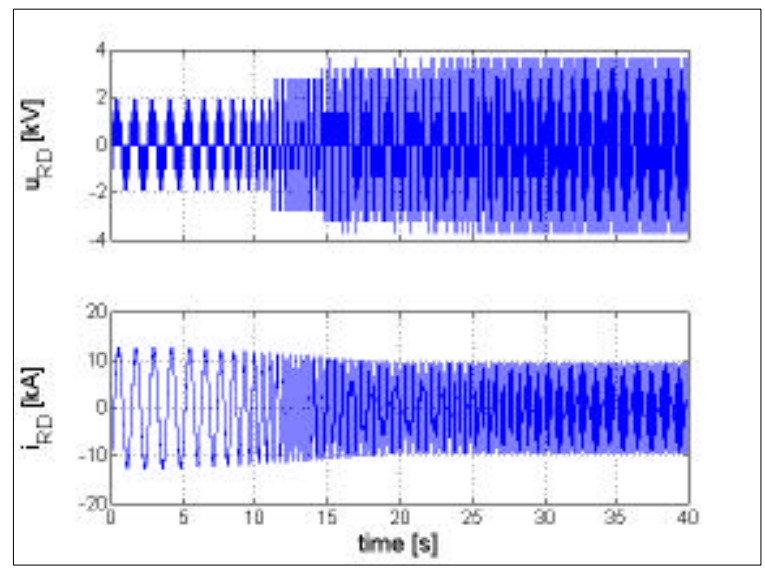

Fig. 11. Rotor voltage and current

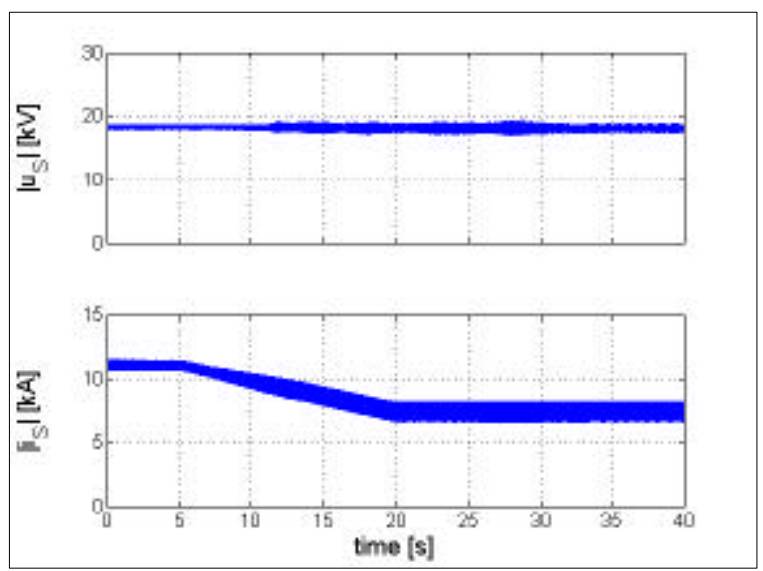

Fig. 12. Amplitudes of stator voltage and current

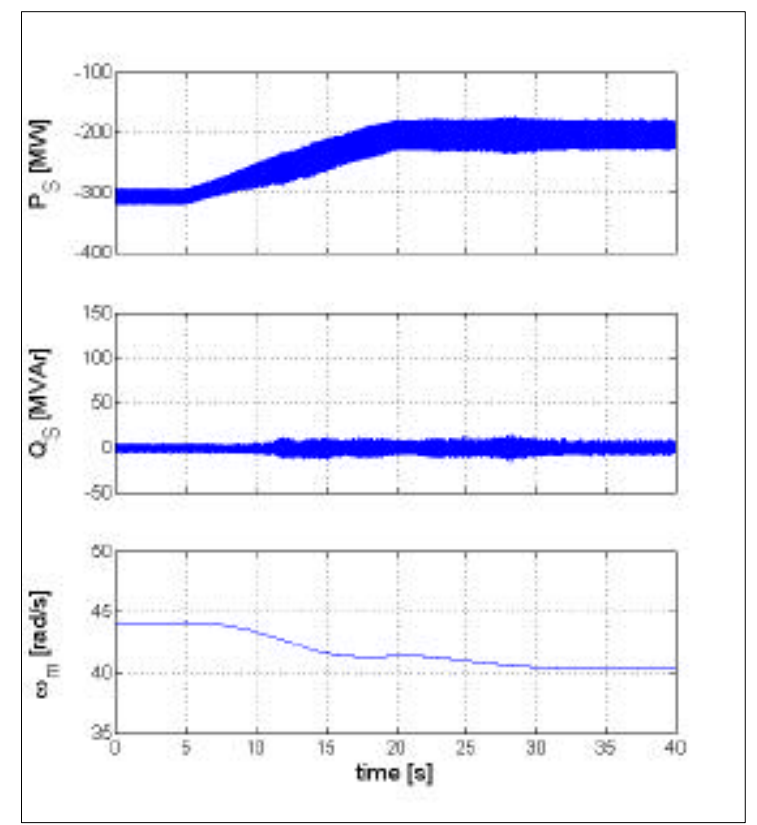

Fig. 13. Active and reactive powers and mechanical speed
Due to the voltage drops in the distribution lines caused by higher current harmonics that flow to the power network, a whole spectrum of higher harmonics arises in the voltage at the machine terminals. This affects the content of higher harmonics in the currents and fluxes of the machine.

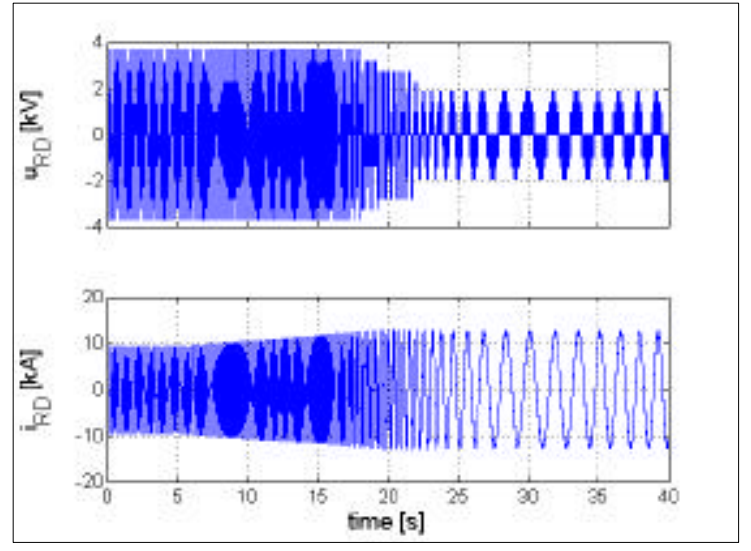

Fig. 14. Rotor voltage and current

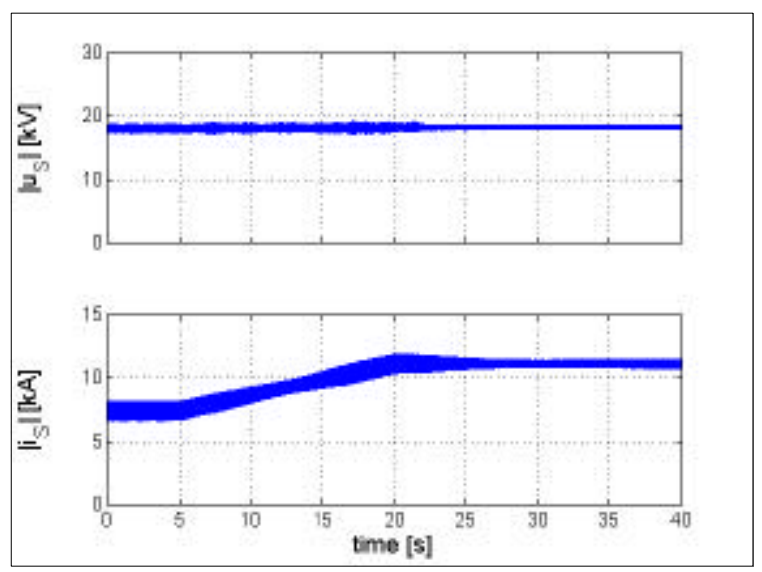

Fig. 15. Amplitudes of stator voltage and current

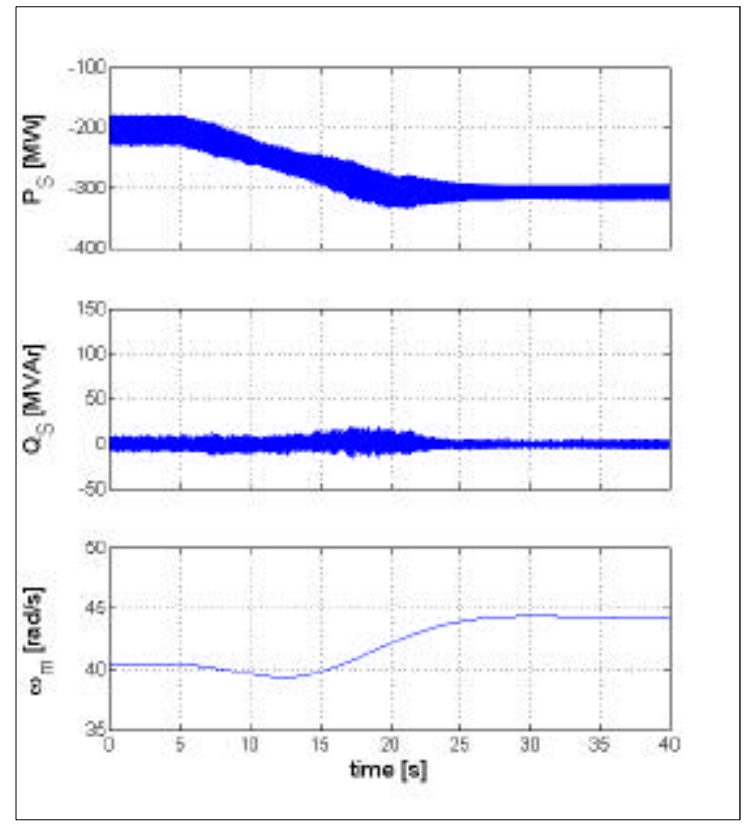

Fig. 16. Active and reactive powers and mechanical speed 
The situation when the demanded generated active power is increased from 200 to $300 \mathrm{MW}$ is illustrated in Figs. 14-16.

The advantage of doubly fed machines is the fact that the kinetic energy of the rotating masses can be utilized temporarily to cover the active power in the grid. At the same time the system speed can drop temporarily, which is not possible with standard synchronous machines. An example of such a drop of speed at simultaneous load increase is shown in Fig. 16.

\section{Conclusions}

The utilization of DFM in power units of medium and low outputs brings some advantages in comparison to the utilization of the standard synchronous machines. They are above all a possibility to control the revolutions and a possibility to control active and reactive outputs by means of a convenient rotor-voltage control. For medium-output machines, a cycloconverter fed from its own transformer is a suitable source of this voltage. Just this complex solution can contribute to higher stability of the power system and to higher quality of electric power. Power units with DFM are advantageous especially for renewable energy sources that means for small hydroelectric power stations. This work solves some problems related to DFM application in power units. Nevertheless, there are some other problems exceeding the range of this work to tackle.

\section{Acknowledgement}

The financial support of the Grant Agency of the Academy of Sciences of the Czech Republic, research grant No. 2057102 is acknowledged.

\section{References}

[1] J. Simond, A. Sapin and D. Schaper, "Expected Benefits of Adjustable Speed Pumped Storage in the European Network". Hydropower into the next century, pp. 579-583, Gmunden, Austria 1999.
[2] P.A. Edvarden, T.F. Nestli, R. Nilsen and H. Kolstad, "Steady-State Power Flow and Efficiency Optimising Analysis of a Variable Speed Constant Frequency Generating System", Proc. of the EPE'97 Conference, Vol. 2, pp. 691-694, Trondheim, Norway, September 1997.

[3] A. Masmoudi, A. Toumi, M. B. A. Kamoun, M. Poloujadoff, "Power Flow Analysis and Efficiency Optimization of a Doubly Fed Synchronous Machine“, Electric Machines and Power Systems, Vol. 21, pp. 473-491, 1993.

[4] L. Schreier, M. Chomát, J. Bendl, "Working Regions of Adjustable-Speed Units with Doubly Fed Machines", Proceedings of IEMDC Conference, Seattle, USA, May 1999.

[5] M. Chomát, L. Schreier, J. Bendl, "Numerical Model of Adjustable-Speed Power Unit Using Doubly Fed Machine with Cycloconverter in Rotor Circuit", Proceedings of 8th European Conference on Power Electronics and Application - EPE'99, Lausanne, Switzerland, 1999.

[6] M. Yamamoto, O. Motoyoshi, "Active and reactive power control for doubly-fed wound rotor induction generator", IEEE Trans. on Power Electronics, Vol. 6, No. 4, pp. 624-629, October 1991.

[7] M. Chomát, J. Bendl, L. Schreier, "Extended Vector Control of Doubly Fed Machine Under Unbalanced Power Network Conditions“, International Conference on Power Electronics Machines and Drives, University of Bath, UK, April 2002.

[8] J. Bendl, M. Chomát and L. Schreier, "Independent Control of Positive- and Negative-Sequence Current Components in Doubly Fed Machine", Procedings of International Conference on Electrical Machines ICEM2002, Brugge, Belgium, 25-28 August, 2002

[9] T. Kuwabara, A. Shibuya, H. Furuta, H. Kita and K. Mitsuhashi, "Design and Dynamic Response Characteristic of 400 MW Adjustable Speed Pumped Storage Unit for Ohkawachi Power Station", IEEE Transactions on Energy Conversion, Vol. 11, No. 2, pp. 376-384, June 1995

[10] T. Kokado, M. Nakazawa, S. Kayukawa and T. Kuwabara, "Operating Experiences with Adjustable Speed Machines Contribution to Systém Stability“, Proc. of the Conference Hydropower into next Century, Austria, pp. 569-578, October 1999. 